\title{
EL DISTRITO 22@BARCELONA COMO ELEMENTO DE RELOCALIZACIÓN DE LAS EMPRESAS DE LA CIUDAD. UN ANÁLISIS DE LAS ANTIGUAS Y LAS NUEVAS UBICACIONES DE LAS SEDES EMPRESARIALES ${ }^{1}$
}

\author{
Daniel Paül i Agustí \\ Departament de Geografia i Sociologia. Universitat de Lleida \\ dpaul@geosoc.udl.cat
}

\section{RESUMEN}

El análisis de las repercusiones de una transformación urbana suele centrarse en el propio espacio transformado. No obstante, los efectos pueden ser más amplios e incluir toda la ciudad. La investigación analiza las repercusiones generadas en la ciudad de Barcelona por el proceso de traslado de las distintas compañías hacía el distrito tecnológico 22@Barcelona. Los resultados apuntan a que la mayoría de empresas se han trasladado des de otras áreas de la ciudad, siendo la llegada de nuevas compañías de otros contextos extremadamente limitada. En este sentido, el nuevo distrito ha contribuido más a una relocalización dentro Barcelona que a un fortalecimiento de su tejido económico. Igualmente, la relocalización ha contribuido a generar efectos en otras áreas de la ciudad, como la pérdida de puestos de trabajo en algunos barrios o cierta turistificación del centro histórico.

Palabras clave: Barcelona, distrito creativo, transformación urbana.

Fecha de recepción: septiembre 2015.

Fecha de aceptación: septiembre 2016.

1 El presente artículo se integra dentro del proyecto financiado por el Plan Nacional de I+D+i "La gestión espacial de la conflictividad social. La ordenación urbana de los espacios colectivos y las morfologías arquitectónicas" (CSO2015-64643-R). También ha sido posible gracias a la financiación del Grup de Recerca Consolidat «Territori i Societat» de la Generalitat de Catalunya (2014 SGR 973). 


\section{ABSTRACT}

The analysis of the urban regeneration projects is usually focused on the impacts achieved in the same urban area. However, in some case the induced effects could be broader, and can affect the whole city. Within this framework, the research presented here analyses the impacts generated in the city of Barcelona by the creation of the 22@Barcelona technology district. The results suggest that there has been a process of transfer of various companies from different parts of the city to the district; while the arrival of new companies from other territories has been extremely limited. In this sense, the new district has contributed more to a relocation or concentration within Barcelona, rather than a strengthening of its economic network. Moreover, this relocation has helped to generate effects in other areas of the city, such as a loss of jobs in certain neighbourhoods or the touristification of the historical centre.

Keywords: Barcelona, creative district, urban transformation.

\section{LA CREACIÓN DE NUEVOS BARRIOS EN ÁREAS URBANAS CONSOLIDADAS}

Las operaciones de transformación física y funcional de áreas obsoletas se han convertido en un elemento habitual de las ciudades occidentales. Desde los años ochenta han sido muchas las ciudades que han apostado por impulsar este tipo de actuaciones. En general se trata de generar un cambio de uso motivado por la obsolescencia de infraestructuras claramente industriales. A modo de ejemplo podemos citar los trabajos realizados sobre las antiguas áreas portuarias de Baltimore, Génova o Bilbao (Gastaldi y Camerin, 2013).

Generalmente los efectos de los cambios que se pueden dar en estos nuevos espacios urbanos se han analizado más desde el punto de vista de la producción de ese espacio que en términos de efectos sobre el conjunto de la ciudad (Paris, 2003). Así, aspectos como la gentrificación, la accesibilidad, el posicionamiento del espacio en el contexto internacional o la especialización de estos nuevos espacios, entre otros, han sido objeto de múltiples publicaciones. Más raros han sido los artículos que se han focalizado en las repercusiones a una escala territorial más amplia. El presente análisis pretende reflexionar sobre las repercusiones que las grandes transformaciones urbanísticas tienen en estos contextos más amplios. Para ello nos basaremos en una actuación urbanísticas ambiciosa: la transformación del antiguo barrio industrial del Poblenou de Barcelona en el distrito tecnológico (22@Barcelona).

No nos centraremos, en consecuencia, en las repercusiones generadas por el 22@Barcelona en su propia área. Este aspecto ya ha sido ampliamente tratado en un buen número de trabajos entre los cuales podemos destacar los que abordan el área desde un punto de vista urbanístico (Boixader, 2005), de la internacionalización de sus empresas (Leon, 2008), del conocimiento (Dot, Casellas y Pallarès, 2010), del posicionamiento internacional de Barcelona (Sabaté y Tironi, 2008), de su patrimonio industrial (Checa, 2007) o de la creatividad (Pareja-Eastaway, 2010), entre muchas otras visiones.

En términos generales las grandes transformaciones urbanas implican una serie de cambios ambiciosos: se planifica, se construye y se quiere crear actividad económica, social y 
a menudo residencial en cortos períodos de tiempo. A modo de ejemplo, en el caso que se presenta, las previsiones son que las transformaciones del área de estudio se realicen en 20 años (2000-2020) (Ajuntament de Barcelona, 2010: 8). Un corto periodo de tiempo en la historia de la ciudad que hace que, a menudo, no se disponga de tiempo suficiente para generar, desde los espacios impulsados, nuevas actividades capaces de dotar con actividad el área. Esto da lugar a que los nuevos espacios se llenen de vecinos, de empresas o de servicios que anteriormente se ubicaban en otros barrios. A nivel de ciudad, por lo menos en las primeras etapas de estos proyectos, no se crea nueva actividad sino que se traslada la actividad que se situaba en otros barrios.

Son varios los trabajos que han descrito esta movilidad asociada a los procesos urbanísticos de transformación. Un ejemplo es la zona del Parque das Nações de Lisboa (Portugal). Esta área alojó la Exposición Mundial de 1998. Las actuaciones derivadas de la Exposición supusieron una mejor en la accesibilidad. Esto hizo más atractiva el área, que se transformó en un nuevo polo de centralidad para empresas e instituciones anteriormente localizadas en la ciudad de Lisboa (Serdoura, 2008: 187).

Un caso similar, con más recorrido histórico, sería el del barrio de la Défense, próximo a París (Francia). La construcción de esta nueva área se inició en los años sesenta. Actualmente es uno de los principales barrios de negocio de Europa hasta el punto que sus infraestructuras, situación y actividad hacen que algunos autores se empiecen a inquietarse de la importancia que está adquiriendo la Défense. Una importancia que podría suponer un debilitamiento del espacio económico de la propia ciudad de París. Así, el mejor posicionamiento de la Défense estaría poniendo en duda la capacidad de París para satisfacer las necesidades de las sedes empresariales (Terral y Padeiro, 2013; Davezies, 2006).

Pese a estos procesos, son pocos los trabajos que entran a analizar qué consecuencias tienen los nuevos distritos para las antiguas zonas de implantación. Un caso en el que sí que se ha valorado es en Melbourne (Australia). En él se muestra como el aumento de la población de los distritos centrales comportó un cambio en la industria. Las actividades de producción, transporte y almacenamiento se desplazaron hacia las periferias, mientras que los espacios centrales pasaron a acoger servicios a empresas (Watkins, 2009: 1562). Una situación similar es la que se dio a False Creek South (Vancouver, Canadá). Su rápido desarrollo como CBD, entre 1977 y 1985, propició la transformación de áreas cercanas, como el West End, para uso residencial (Hutton, 2004: 1960) y también generó una erosión de los barrios obreros tradicionales y la rápida descentralización industrial. Un hecho que generó un debate sobre políticas locales que llevó a la creación de una Industrial Development Office y un trabajo posterior para revitalizar el centro de la ciudad y conseguir un nuevo mix de actividades (Hutton, 2004: 1961-1964). Un último ejemplo en la misma línea es el de Bruselas (Bélgica). A partir de la segunda mitad del siglo XX una serie de antiguos barrios residenciales fueron substituidos por barrios de negocios (con el ilustrativo nombre de Plan Manhattan) y centros administrativos (estatales y europeos). Estos desarrollos han supuesto que "Bruselas no sabe qué hacer de sus oficinas vacías (1'5 millones de $\left.\mathrm{m}^{2}\right)(\ldots)$ las nuevas oficinas son alquiladas sistemáticamente por empresas públicas, paraestatales o locales, vaciando otros edificios de oficinas de otras partes de la ciudad" (Tomson, 2015). La ciudad consolidada tiende, mediante estos procesos, a perder una serie de funciones, que pasan a las nuevas áreas. 
El traslado de las actividades económicas tiende a reforzar centros o subcentros y es uno de los motivos por los que las áreas económicas, sociales o culturales de las diversas ciudades no son fijos (Hillier, 2001: 02.25). No obstante, en etapas históricas anteriores, la creación de nuevas áreas tendía a hacerse para acoger actividad del sector industrial, que había perdido funcionalidad en el espacio central. Una vez liberado, el espacio central se ocupaba con nuevas actividades, mayoritariamente terciarias (Carrincazeaux, 2000). Este es el caso de Barcelona, que apostó por las industrias creativas como elemento articulador de la transformación de una parte del barrio industrial del Poblenou.

El presente artículo se centrará en analizar si en el caso de la actuación urbanística de Barcelona se mantienen los efectos descritos anteriormente. La principal diferencia con estos casos radica en que en el proceso de planificación de Barcelona se era consciente de las debilidades descritas, por lo que la planificación apostaba por crear un nuevo distrito creativo que incluyese "un proyecto de renovación urbana y un nuevo modelo de ciudad que quiere dar respuesta a los retos de la sociedad del conocimiento"2. La planificación de la operación se pensó, en consecuencia, en términos de ciudad. Se planeó pensando en beneficiar y potenciar la imagen del conjunto de Barcelona como ciudad abierta a la sociedad del conocimiento. Ahora bien, varios cambios en la gestión del proyecto y la coincidencia del desarrollo del plan con la crisis económica obligaron a reformular algunos aspectos de su gestión. Los objetivos iniciales del plan se vieron afectados y la ocupación del distrito acabó generando efectos en otras áreas de la ciudad, pero no siempre en la línea de lo inicialmente previsto. Es en este aspecto que se focalizará el artículo. Para ello se partirá del análisis de las principales actividades instaladas en el área 22@Barcelona, su procedencia y los motivos que llevaron las distintas compañías a instalarse en el área.

\section{METODOLOGÍA}

Con fecha junio de 2015 había en el distrito 22@Barcelona un total de 8.223 compañías para un volumen total de 93.000 trabajadores estimado (GAPS, 2015: 12). Ahora bien, la fragmentación de las compañías presentes era elevada: cincuenta compañías concentraban un tercio de los trabajadores. Además los datos del mismo estudio señalaban que la concentración de los trabajadores en grandes empresas aumentaba. En el año 2010 el $42 \%$ de los trabajadores del distrito se encontraban en empresas con más de 50 trabajadores, mientras que en 2015 eran el 58\% (GAPS, 2015: 22). Esta concentración llevó a focalizar el presente trabajo en las empresas medianas y grandes (aquellas con más de 50 trabajadores), ya que se consideró que eran las que generarían mayores repercusiones tanto en el conjunto de la ciudad como en el distrito.

Para identificar el listado de las compañías del distrito con más de 50 trabajadores recurrimos a la base de datos SABI (Sistema de Análisis de Balances Ibéricos). Una base que recopila información de más de un millón de empresas españolas a partir de fuentes oficiales como los Registros Mercantiles. Esta base de datos identificó 120 empresas activas en el área con más de 50 trabajadores.

2 http://www.22barcelona.com/content/blogcategory/49/280/lang,es/ (consultado el 06/08/2015). 
A partir de la identificación de las empresas se procedió a recopilar información sobre sus posibles traslados. Para ello se vaciaron varias publicaciones oficiales dedicadas a informar de los avances del proyecto de transformación urbana. En ellas se informaba, entre otros temas, de las implantaciones de nuevas empresas en el área. Concretamente se trataba, en primer lugar, de la agenda de noticias de la sociedad municipal 22 ARROBA BCN, S.A.U, encargada de la transformación del distrito ${ }^{3}$. En segundo lugar, Barcelona Growth ${ }^{4}$, revista editada por Barcelona Activa, organización municipal responsable de impulsar el desarrollo económico del conjunto de la ciudad. En ambos casos se analizaron, mediante palabras clave, el conjunto de información para identificar las compañías trasladadas y se cruzaron los datos obtenidos con los nombres y referencias de la base SABI.

Para verificar la fiabilidad de los datos se realizaron 7 entrevistas presenciales a varios técnicos de la oficina municipal encargada de la gestión de la transformación del distrito y miembros de asociaciones vecinales del barrio. En ninguna de las entrevistas se citó como trasladada al barrio ninguna empresa que no constase en el vaciado anterior, lo que reforzó la fiabilidad de las fuentes.

Cuando la información de la base de datos y de los medios analizados no era suficiente para completar la información requerida para el presente artículo se recurrió a otras fuentes, como periódicos y webs corporativas. Este medio se usó básicamente para localizar direcciones de las antiguas sedes y sus metros cuadrados, que no aparecían en los medios apuntados anteriormente.

Así mismo se contactó telefónicamente con las distintas compañías analizadas para recopilar información de carácter más cualitativo (motivaciones del traslado, experiencia, etc.). Se obtuvieron 29 respuestas válidas. Una cifra relativamente baja dada la dificultad para acceder, en las grandes empresas, a personas con los datos deseados, pero que permite obtener un buen muestreo de las características de las empresas presentes en el área.

Finalmente, para analizar los usos actuales de las antiguas sedes se visitaron físicamente todas las localizaciones en febrero de 2014. El análisis del conjunto de información permitió identificar 67 empresas con más de 50 trabajadores trasladadas al distrito des del año 2000 hasta 2014. Fue sobre estas empresas sobre las que nos focalizamos.

\section{LA EVOLUCIÓN DEL BARRIO DEL POBLENOU: DE ESPACIO INDUSTRIAL A DISTRITO DE LA INNOVACIÓN (22@BARCELONA)}

El barrio del Poblenou es un área de implantación histórica situada al nordeste del centro histórico de Barcelona, en el distrito de Sant Martí. Desde el siglo XVIII el Poblenou había empezado a concentrar algunas industrias. Un proceso que se intensificará en el siglo XIX. La presencia industrial se mantuvo hasta los años setenta. Posteriormente los cambios experimentados por la industria comportaron una etapa de crisis. No obstante, de forma prácticamente simultánea, comenzaron a aparecer los primeros proyectos de renovación del barrio. Estos proyectos se concentran en un primer momento en las periferias del área.

3 http://www.22barcelona.com/content/blogcategory/23/90/lang,ca/ (consultado en enero de 2014).

4 http://w42.bcn.cat/web/en/index.jsp (consultado en enero de 2014). 
El primero paso fue la construcción de la Villa Olímpica de los Juegos Olímpicos de 1992. Este proceso se inició en la zona litoral más cercana al centro de la ciudad. La actuación transformó $720.000 \mathrm{~m}^{2}$ del litoral barcelonés, que pasó de estar dedicado mayoritariamente a la industria a usos residenciales y de ocio (recuperación de las playas para los ciudadanos). A finales de los años noventa, también en la zona más cercana al centro de la ciudad, pero hacia el interior, se construyeron algunos equipamientos, como el Teatro Nacional de Cataluña (inaugurado en 1997) o el Auditorio de Barcelona (1999), que intentaron crear una nueva centralidad urbana. Al mismo tiempo, se inició el gran proceso de reforma de la prolongación de la Avenida Diagonal hasta el mar. La urbanización del sector Diagonal Mar, último vértice del triángulo, de cara a la celebración en 2004 del Fòrum Universal de les Cultures terminó de modificar la configuración de los sectores más periféricos del barrio.

De forma paralela se inició la renovación de la parte central del Poblenou. Este espacio aún estaba ocupado por naves industriales, tanto en uso como abandonadas. La transformación la gestionaría una sociedad mercantil íntegramente municipal y el proyecto se conocería como 22@Barcelona (figura 1). El objetivo del cambio era crear "las condiciones y los incentivos adecuados para favorecer la renovación urbana del sector y la localización de nuevos puestos de trabajo cualificados y de actividades productivas intensivas en conocimiento" (Ajuntament de Barcelona, 2010: 8).

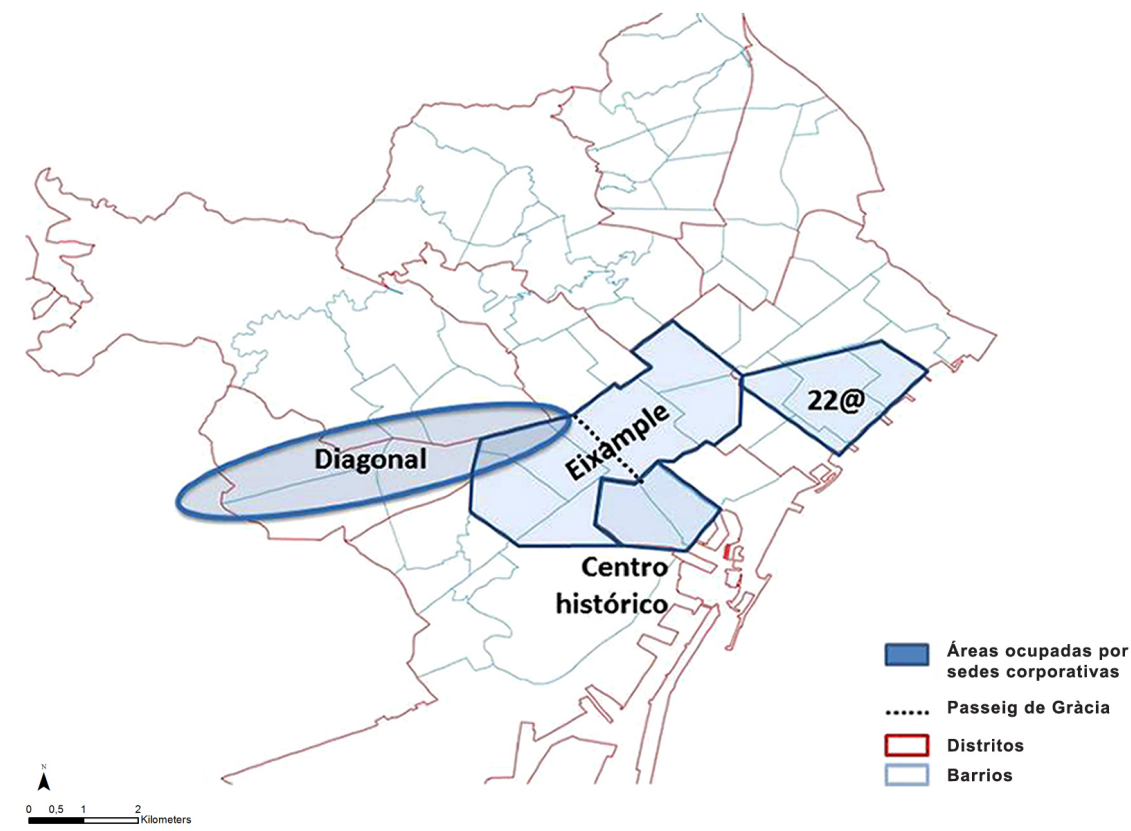

Fuente: Autor. Cartografía: Ajuntament de Barcelona. 
Los cambios más visibles se concentraron en los aspectos urbanísticos: intensificación de la edificabilidad de la nueva subzona, creación de nuevos espacios para equipamientos, reurbanización completa del sector y creación de mecanismos de planeamiento derivado para desarrollar la transformación.

Las previsiones eran actuar sobre una superficie de 198'26 Ha, transformando 1.159.626 $\mathrm{m}^{2}$ de suelo. En otros términos, la actuación aspiraba a transformar el $2 \%$ del término municipal de Barcelona. No obstante, el proyecto iba más allá de la transformación física y la creación de un distrito de negocios. La intención era crear una ciudad compacta y diversa, que evitara un modelo de especialización territorial de usos. En este sentido se preveía la construcción de viviendas (4.000 de ellas de protección oficial), la creación de nuevos campus universitarios, la dotación de equipamientos (145.000 $\mathrm{m}^{2}$ de suelo) y la creación de nuevas zonas verdes $\left(114.000 \mathrm{~m}^{2}\right)$. En este sentido, pese al peso de las actuaciones físicas, la actuación se enmarcaba dentro de un conjunto de actuaciones más amplias, que pretendían reforzar el papel de Barcelona como Smart City. Por este motivo las actuaciones previstas no se limitaron únicamente al ámbito puramente económico, sino que incluyeron actuaciones en aspectos como el verde urbano, la inclusión social, la ciencia y tecnología, la vivienda, la movilidad o la calidad de vida (Tuba, Almirall y Wareham, 2013, 141).

\section{UNA TRANSFORMACIÓN AMBICIOSA}

Inicialmente la transformación física del barrio se desarrolló a un ritmo muy rápido; el desarrollo inicial del proyecto coincide con los años de fuerte crecimiento de la construcción en la economía española. Entre los años 2000 y 2008 se concedieron licencias en el distrito para actuar en 3' 6 millones de $\mathrm{m}^{2}$ (figura 2). No disponemos de datos a nivel detallado del área del 22@Barcelona, pero por el contexto podemos considerar que la mayor parte de las actuaciones desarrolladas en el distrito de Sant Martí correspondían a la zona del22@ Barcelona ${ }^{5}$. Una buena muestra de la intensidad de la transformación física de este espacio es que en el conjunto del período 2000-2014 el distrito, con una superficie que representa el 10 ’3\% de la de Barcelona, concentró el 23'1\% del uso de techo de las licencias concedidas en el conjunto de la ciudad. Las cifras máximas se consiguieron en el período 2005-2008, llegándose en 2006 a más de medio millón de metros cuadrados concedidos, lo que representaba un 31'6\% de las licencias de la ciudad. Posteriormente, entre 2009 y 2014, la actividad se ha moderado (Paül, 2014b). Pese a ello, en este período, se han concedieron licencias en el distrito para actuar en casi $800.000 \mathrm{~m}^{2}$, lo que representa el $18,5 \%$ de las licencias concedidas en la ciudad.

Los datos anteriores nos señalan una actuación importante en el campo urbanístico. Por ámbitos, el más destacado fue el residencial (30'7\% del total de $\mathrm{m}^{2}$ de uso de planta concedidos), seguido de los aparcamientos (27'5\%) y las oficinas (19'2\%). Destaca, asimismo, que las licencias para usos industriales sólo representaron un 1'3\% de la superficie concedida.

5 Los datos referentes a superficie de techo previsto total según usos por distritos apuntan que entre los años 2000 y 2009 los metros cuadrados renovados en el conjunto del distrito de Sant Martí fueron de $3.880 .000 \mathrm{~m}^{2}$. En el mismo periodo, la memoria del22@señala que se renovaron en esta área un total de 2.830.000m² (Ajuntament de Barcelona, 2010, p.13). Así, podemos concluir que aproximadamente el 73\% de los metros cuadrados renovados del área de Sant Martí corresponden a la zona del22@. 
Estos datos nos demuestran la intensidad y la dirección del cambio experimentado en la zona. Si comparamos los datos del distrito con los de Barcelona, observamos que el 56'7\% de la superficie de techo de las licencias concedidas por oficinas en toda Barcelona se concentraron en Sant Martí (es decir, mayoritariamente en el 22@Barcelona).

Figura 2

EVOLUCIÓN DE LA SUPERFICIE DE TECHO DE LAS LICENCIAS CONCEDIDAS SEGÚN USO DE LA PLANTA (M²). DISTRITO DE SANT MARTI

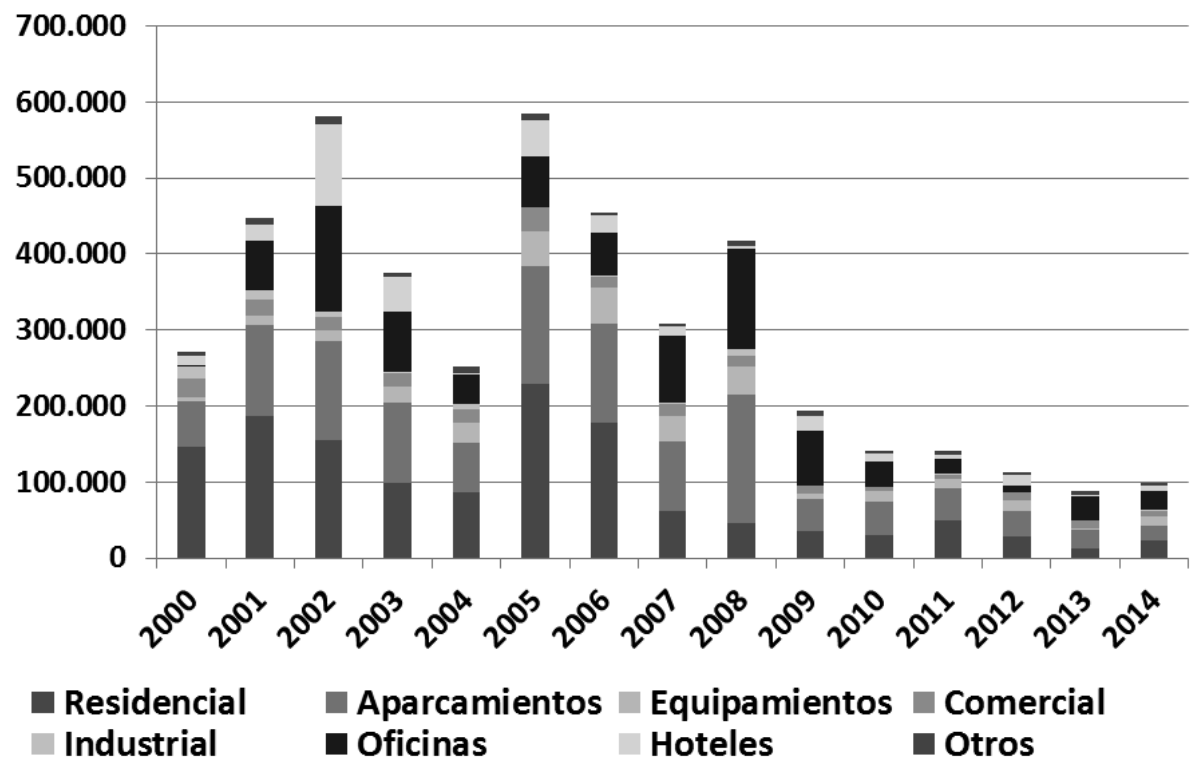

Fuente: elaboración propia con datos de www.bcn.cat/estadistica (consultado el 22 julio 2015).

\section{DESPLAZAMIENTOS DE SEDES EMPRESARIALES RELACIONADAS CON EL 22@BARCE- LONA}

En pocos años el paisaje urbano de viejas fábricas medio derruidas se cambió por nuevos edificios de oficinas y hoteles (Paül, 2014a). Ahora bien, en su mayoría no se trataba de creación de nueva actividad, sino de traslados. Un dato interesante a resaltar es que un $41 \%$ de las compañías presentes en el área analizada procedían de otros espacios (GAPS, 2015). Una cifra nada despreciable y que contribuyó a modificar, además del barrio, ciertos espacios del conjunto de la ciudad, aspecto sobre el cual nos centraremos.

A continuación presentamos una breve descripción de las principales compañías que se han instalado en el distrito, del lugar de origen del traslado y de algunos datos que pueden ayudar a contextualizar las repercusiones generadas. Para un mejor análisis hemos organizado las compañías en función del uso actual de los antiguos espacios ocupados antes del traslado al 22@Barcelona. La fecha de referencia es febrero de 2014. 


\section{V.1. Antiguas sedes centrales sin uso}

En algunos de los casos analizados las antiguas sedes han quedado sin uso. Esta casuística se da cuando una compañía ocupaba todo el inmueble, generalmente fuera de las áreas turísticas o empresariales de la ciudad (tabla 1).

Tabla 1

COMPAÑÍAS TRASLADADAS AL 22@BARCELONA LAS ANTIGUAS SEDES DE LAS CUALES ESTÁN DESOCUPADAS

\begin{tabular}{|c|c|c|c|c|c|c|}
\hline Nombre & $\begin{array}{c}\text { Sector de } \\
\text { actividad }\end{array}$ & $\begin{array}{c}\text { Localización de } \\
\text { la antigua sede }\end{array}$ & $\begin{array}{c}\text { Situación } \\
\text { de la nueva } \\
\text { sede }\end{array}$ & $\begin{array}{c}\text { Año } \\
\text { traslado }\end{array}$ & $\begin{array}{c}\text { Superficie } \\
\text { nueva sede }\end{array}$ & $\begin{array}{c}\text { Trabaja- } \\
\text { dores }\end{array}$ \\
\hline Konecta & $\begin{array}{c}\text { Marketing } \\
\text { telefónico }\end{array}$ & $\begin{array}{c}\text { Proximidad del } \\
\text { centro histórico }\end{array}$ & $\begin{array}{c}\text { C/ Llacuna } \\
56-70\end{array}$ & 2013 & $3.000 \mathrm{~m}^{2}$ & 500 \\
\hline Liberty Seguros & Seguros & $\begin{array}{c}\text { Periferia del 22@ } \\
\text { Barcelona }\end{array}$ & $\begin{array}{c}\text { C/ Llacuna } \\
56-70\end{array}$ & 2012 & $5.500 \mathrm{~m}^{2}$ & Sin datos \\
\hline RBA & Editorial & $\begin{array}{c}\text { Varias sedes. Las } \\
\text { principales en la } \\
\text { zona norte de la } \\
\text { ciudad. }\end{array}$ & $\begin{array}{c}\text { Avinguda } \\
\text { Diagonal, } \\
189\end{array}$ & 2009 & $25.000 \mathrm{~m}^{2}$ & 1.000 \\
\hline $\begin{array}{c}\text { Schneider } \\
\text { Electric }\end{array}$ & Energía & $\begin{array}{c}\text { Proximidad del } \\
\text { centre histórico }\end{array}$ & $\begin{array}{c}\text { C/ Badajoz, } \\
145\end{array}$ & 2008 & $10.500 \mathrm{~m}^{2}$ & Sin datos \\
\hline Telefónica & Comunicación & Eixample & $\begin{array}{c}\text { Avinguda } \\
\text { Diagonal, } 00\end{array}$ & 2011 & $34.000 \mathrm{~m}^{2}$ & 1.200 \\
\hline
\end{tabular}

Fuente: elaboración propia.

Pese a tratarse de relativamente pocos casos no podemos ignorar las repercusiones generadas. Las cinco antiguas sedes analizadas representaban, en su conjunto, una superficie aproximada de $70.000 \mathrm{~m}^{2}$ y estimamos que daban trabajo a más de 3.000 personas. Unas cifras nada despreciables. En consecuencia, los traslados han generado algunos vacíos urbanos importantes. Por ejemplo, la sede de Telefónica ocupaba una manzana entera. De hecho, este ha sido un caso paradigmático de proyecto afectado por la burbuja inmobiliaria española. El edificio fue comprado por una sociedad de capital riesgo por 185 millones de euros en 2007. La intención era que el edificio se transformara en $38.000 \mathrm{~m}^{2}$ de viviendas y 4.000 $\mathrm{m}^{2}$ de espacios comerciales. No obstante, la sociedad compradora entró en quiebra. Finalmente, en el año 2014 el edificio cambió de manos por 56 millones de euros. En medio, Telefónica se trasladó al 22@Barcelona en 2011. Este período de tres años en el que el edificio ha permanecido abandonado ha afectado los alrededores. El edificio ha pasado de ser un punto de referencia y un centro de atracción a escala urbana a una fuente de problemas (ocupaciones ilegales, robos o problemas de falta de mantenimiento). El ejemplo del edificio de Telefónica es muy ilustrativa del perfil de la mayoría de actuaciones: una operación inmobiliaria, relacionada con la recalificación de la antigua sede, es la que motiva el traslado. Un elemento sobre el que volveremos más adelante.

6 Se entiende por proximidad del centro histórico aquellos espacios situados dentro de un radio de 500 metros del distrito de Ciutat Vella. 


\section{V.2. Antiguas sedes que siguen acogiendo oficinas}

Una segunda casuística observada es el cambio prácticamente inmediato de los inquilinos de las sedes trasladadas. En la mayoría de casos las oficinas que se situarían en esta categoría no ocupan grandes espacios. Habitualmente eran compañías que alquilaban algunas plantas en edificios de oficinas compartidos. Los casos en los que se ocupaban más superficie (más de $1.000 \mathrm{~m}^{2}$ ) los hemos recopilado en la tabla 2 .

Tabla 2

COMPAÑIIAS TRASLADADAS AL 22@BARCELONA LAS ANTIGUAS SEDES DE LAS CUALES SIGUEN OCUPADAS?

\begin{tabular}{|c|c|c|c|c|c|c|}
\hline Nombre & $\begin{array}{c}\text { Sector de } \\
\text { actividad }\end{array}$ & $\begin{array}{c}\text { Localización de } \\
\text { la antigua sede }\end{array}$ & $\begin{array}{c}\text { Situación de la } \\
\text { nueva sede }\end{array}$ & $\begin{array}{c}\text { Año } \\
\text { traslado }\end{array}$ & $\begin{array}{c}\text { Superficie } \\
\text { nueva sede }\end{array}$ & $\begin{array}{c}\text { Trabaja- } \\
\text { dores }\end{array}$ \\
\hline Netcentric & $\begin{array}{c}\text { Web content } \\
\text { manegement }\end{array}$ & Port de Barcelona & $\begin{array}{c}\text { Avinguda } \\
\text { Diagonal, } 123\end{array}$ & 2013 & $1.000 \mathrm{~m}^{2}$ & 100 \\
\hline Ogilvy y Mather & $\begin{array}{c}\text { Agencia de } \\
\text { comunicación }\end{array}$ & Diagonal & C/ Bolívia, 68 & 2011 & $4.500 \mathrm{~m}^{2}$ & 60 \\
\hline Vodafone & Telefónica & Diagonal & $\begin{array}{c}\text { Avinguda } \\
\text { Diagonal, } 123\end{array}$ & 2012 & $3.000 \mathrm{~m}^{2}$ & 300 \\
\hline
\end{tabular}

Fuente: elaboración propia.

El traslado de las distintas compañías contribuyó a centralizar oficinas antes repartidas por varios espacios de la ciudad. El fenómeno se ha dado principalmente a través de la construcción de nuevas sedes en el 22@Barcelona. Las empresas localizadas en otras áreas también se han beneficiado puntualmente de estos traslados. Compañías presentes en el mismo edificio, con necesidades de espacio, han visto en el traslado de compañías vecinas una oportunidad para ampliar sus dependencias.

\section{V.3. Antiguas sedes que han cambiado de uso.}

En algunos casos, generalmente en aquellos situados en los espacios más próximos a los grandes nodos turísticos de Barcelona, los antiguos locales han cambiado de uso. Como se puede ver en la tabla 3 , varios espacios han dejado de acoger oficinas de empresas para alojar nuevas actividades.

En términos generales las antiguas sedes de este grupo tienen varios puntos en común. En primer lugar, se trata generalmente de sedes que ocupaban superficies amplias. Igualmente, en muchos casos, las antiguas sedes aprovechaban edificios monumentales con valor histórico. Finalmente destaca, muy probablemente en relación con el factor anterior, que en la mayoría de casos las nuevas actividades instaladas son culturales o educativas.

7 La tabla únicamente incorpora, por motivos de espacio, la información de las empresas con una superficie de más de $1.000 \mathrm{~m}^{2}$. Pese a ello, todos los casos de este grupo han sigo cartografiadas en la figura 3. 
Tabla 3

COMPAÑIIAS TRASLADADAS AL 22@BARCELONA LAS ANTIGUAS SEDES DE LAS CUALES HAN CAMBIADO DE USO

\begin{tabular}{|c|c|c|c|c|c|c|c|}
\hline Nombre & $\begin{array}{l}\text { Sector de } \\
\text { actividad }\end{array}$ & $\begin{array}{c}\text { Situación } \\
\text { antigua } \\
\text { sede }\end{array}$ & $\begin{array}{c}\text { Uso de la } \\
\text { nueva sede }\end{array}$ & $\begin{array}{c}\text { Situación } \\
\text { de la nova } \\
\text { sede }\end{array}$ & $\begin{array}{c}\text { Año } \\
\text { traslado }\end{array}$ & $\begin{array}{c}\text { Superficie } \\
\text { nueva } \\
\text { sede }\end{array}$ & $\begin{array}{c}\text { Trabaja- } \\
\text { dores }\end{array}$ \\
\hline Abacus & $\begin{array}{c}\text { Cooperativa } \\
\text { de venta } \\
\text { de libros y } \\
\text { papelería }\end{array}$ & Eixample & $\begin{array}{l}\text { Tienda de } \\
\text { moda }\end{array}$ & C/ Perú, 186 & 2009 & $2.100 \mathrm{~m}^{2}$ & 100 \\
\hline Altran & $\begin{array}{l}\text { Consultora } \\
\text { tecnológica }\end{array}$ & $\begin{array}{l}\text { Norte de la } \\
\text { ciudad }\end{array}$ & $\begin{array}{l}\text { Bufete de } \\
\text { abogados }\end{array}$ & $\begin{array}{c}\text { C/ Llacuna } \\
56-70\end{array}$ & 2011 & $3.500 \mathrm{~m}^{2}$ & 600 \\
\hline Barcelona TV & Televisión & $\begin{array}{c}\text { Centro } \\
\text { histórico }\end{array}$ & $\begin{array}{c}\text { Escuela } \\
\text { de medios } \\
\text { audiovisuales }\end{array}$ & $\begin{array}{c}\text { Plaça } \\
\text { Tísner, } 1\end{array}$ & 2004 & $3.000 \mathrm{~m}^{2}$ & 150 \\
\hline $\begin{array}{c}\text { Campus de } \\
\text { comunicació } \\
\text { - Universitat } \\
\text { Pompeu Fabra } \\
\text { (UPF) }\end{array}$ & Universidad & $\begin{array}{c}\text { Centro } \\
\text { histórico }\end{array}$ & $\begin{array}{l}\text { Escuela de } \\
\text { diseño y } \\
\text { espacio sin } \\
\text { uso }\end{array}$ & $\begin{array}{c}\text { Roc } \\
\text { Boronat, } \\
138\end{array}$ & 2008 & $28.500 \mathrm{~m}^{2}$ & $\begin{array}{c}3.500 \\
\text { (alumnos, } \\
\text { profesores } \\
\text { y personal } \\
\text { de } \\
\text { servicio) }\end{array}$ \\
\hline $\begin{array}{c}\text { Foment } \\
\text { de les Arts } \\
\text { Decoratives }\end{array}$ & $\begin{array}{c}\text { Asociación } \\
\text { de impulso al } \\
\text { diseño y artes } \\
\text { decorativas }\end{array}$ & $\begin{array}{c}\text { Centro } \\
\text { histórico }\end{array}$ & Museo & $\begin{array}{c}\text { Plaça de } \\
\text { les Glòries } \\
\text { Catalanes, } \\
37\end{array}$ & 2013 & $1.200 \mathrm{~m}^{2}$ & 32 \\
\hline $\begin{array}{c}\text { Grup } \\
\text { Enciclopèdia } \\
\text { Catalana }\end{array}$ & Editorial & Eixample & $\begin{array}{l}\text { Fundación } \\
\text { Mapfre }\end{array}$ & $\begin{array}{l}\text { C/ Josep } \\
\text { Pla, } 95\end{array}$ & 2005 & $3.500 \mathrm{~m}^{2}$ & 100 \\
\hline $\begin{array}{l}\text { Museu de } \\
\text { les Arts } \\
\text { Decoratives } \\
\text { i Museu de } \\
\text { Ceràmica } \\
\end{array}$ & Museo & Diagonal & $\begin{array}{c}\text { Sede de la } \\
\text { Unión para el } \\
\text { Mediterráneo }\end{array}$ & \multirow{2}{*}{$\begin{array}{c}\text { Plaça de } \\
\text { les Glòries } \\
\text { Catalanes, } \\
37\end{array}$} & \multirow[t]{2}{*}{2012} & \multirow[t]{2}{*}{$27.300 \mathrm{~m}^{2}$} & \multirow[t]{2}{*}{ - } \\
\hline $\begin{array}{l}\text { Museu Tèxtil i } \\
\text { d'Indumentària }\end{array}$ & Museo & $\begin{array}{c}\text { Centro } \\
\text { histórico }\end{array}$ & $\begin{array}{c}\text { Museo } \\
\text { culturas del } \\
\text { mundo }\end{array}$ & & & & \\
\hline $\begin{array}{c}\text { RTVE y } \\
\text { Agencia EFE }\end{array}$ & $\begin{array}{c}\text { Radio y } \\
\text { comunicación }\end{array}$ & $\begin{array}{l}\text { Alrededor } \\
\text { del centro } \\
\text { histórico }\end{array}$ & $\begin{array}{c}\text { Tienda Apple } \\
\text { y oficinas }\end{array}$ & $\begin{array}{c}\text { Roc } \\
\text { Boronat, } \\
127\end{array}$ & 2008 & $3.000 \mathrm{~m}^{2}$ & 150 \\
\hline
\end{tabular}

Fuente: elaboración propia.

Entre los ejemplos anteriores destaca el caso del Foment de les Arts i el Disseny (FAD). Una histórica asociación creada en 1903 que en 2013 trasladó, por primera vez en más de un siglo de existencia, la sede de la asociación fuera del centro histórico de la ciudad. El motivo fue el incremento de los costes del alquiler. Actualmente la antigua sede se encuentra sin uso, pero existe el proyecto que acoja la ampliación del museo MACBA.

Un caso específico de cambio de uso en las antiguas sedes es el de su transformación en hotel. A pesar de ser relativamente pocos casos, no dejan de ser significativos de un cambio 
de usos en un determinado espacio: el centro histórico. De hecho, los cuatro casos identificados (tabla 4), se encuentran distantes entre sí unos 1.500 metros. La transformación supone un cambio total en el uso. Antiguos edificios de oficinas, en algunos casos dedicados a la atención a los ciudadanos, cambian a usos turísticos.

Tabla 4

COMPAÑIIAS TRASLADADAS AL 22@BARCELONA CON ANTIGUAS SEDES TRANSFORMADAS EN HOTEL

\begin{tabular}{|c|c|c|c|c|c|c|}
\hline Nombre & $\begin{array}{l}\text { Sector de } \\
\text { actividad }\end{array}$ & $\begin{array}{c}\text { Situación de } \\
\text { la antigua } \\
\text { sede seu }\end{array}$ & Hotel & $\begin{array}{c}\text { Año } \\
\text { apertura }\end{array}$ & $\begin{array}{c}\text { Año } \\
\text { traslado }\end{array}$ & $\begin{array}{l}\text { Situación de } \\
\text { la nueva sede }\end{array}$ \\
\hline $\begin{array}{c}\text { Cuatrecasas } \\
\text { Gonçalves } \\
\text { Pereira }\end{array}$ & Abogados & $\begin{array}{l}\text { Eixample / } \\
\text { Diagonal }\end{array}$ & $\begin{array}{c}\text { Four } \\
\text { Seasons }\end{array}$ & Previsto & 2017 & - \\
\hline Henkel & $\begin{array}{l}\text { Productos de } \\
\text { limpieza }\end{array}$ & $\begin{array}{c}\text { Proximidad } \\
\text { del centro } \\
\text { histórico }\end{array}$ & $\begin{array}{l}\text { Meridià } \\
\text { Capital }\end{array}$ & Previsto & 2015 & Bilbao, 72 \\
\hline Telefónica & Comunicación & $\begin{array}{c}\text { Proximidad } \\
\text { del centro } \\
\text { histórico }\end{array}$ & $\begin{array}{c}\text { Catalonia } \\
\text { Square, } \\
4 *\end{array}$ & 2011 & 2014 & $\begin{array}{c}\text { Avda. } \\
\text { Diagonal } 00\end{array}$ \\
\hline Torraspapel & $\begin{array}{l}\text { Sector del } \\
\text { papel }\end{array}$ & $\begin{array}{l}\text { Proximidad } \\
\text { del centro } \\
\text { histórico }\end{array}$ & $\begin{array}{c}\text { Grupotel } \\
\text { Gran Via } \\
678,4^{*}\end{array}$ & 2008 & 2008 & C/ Llull, 331 \\
\hline
\end{tabular}

Fuente: elaboración propia.

Esta tipología de cambios parece que continuarán en los próximos años no sólo con las sedes de las compañías. A modo de ejemplo, el Gobierno de Cataluña planea centralizar varias dependencias en unas nuevas instalaciones situadas en la periferia de la ciudad (Zona Franca). Todo parece indicar que algunas de las actuales sedes se podrían transformar, si la normativa municipal lo permite, en hotel o equipamiento turístico.

\section{V.4. Atracción de compañías de fuera de la ciudad de Barcelona}

Un último aspecto que queda por reseñar es que la mayoría de compañías analizadas instaladas en el distrito 22@Barcelona procedían de la ciudad de Barcelona. Sólo hemos encontrado unos pocos casos en los que el nuevo distrito ha atraído compañías medianas o grandes implantadas anteriormente en otras localidades, generalmente muy cercanas a Barcelona (tabla 5).

La información anterior apuntaría que la capacidad del 22@Barcelona para captar actividad económica hacia Barcelona ha sido limitada. En este sentido, los datos analizados refuerzan el hecho de que uno de los principales pilares de crecimiento del 22@Barcelona ha sido la captación de actividades ya instaladas en otros barrios de Barcelona, con las distintas repercusiones descritas. 
Tabla 5

COMPAÑÍAS TRASLADADAS AL 22@BARCELONA DES DE FUERA DE BARCELONA

\begin{tabular}{|c|c|c|c|c|c|}
\hline Nombre & $\begin{array}{c}\text { Sector de } \\
\text { actividad }\end{array}$ & $\begin{array}{c}\text { Localidad } \\
\text { antigua sede }\end{array}$ & $\begin{array}{c}\text { Distancia a } \\
\text { Barcelona }\end{array}$ & Nueva sede & $\begin{array}{c}\text { Año } \\
\text { traslado }\end{array}$ \\
\hline Emergia & $\begin{array}{c}\text { Comunicación } \\
\text { en red }\end{array}$ & $\begin{array}{c}\text { l'Hospitalet de } \\
\text { Llobregat }\end{array}$ & $6 \mathrm{~km}$ & $\mathrm{C} /$ Àvila, 60-64 & 2011 \\
\hline $\begin{array}{c}\text { Internet } \\
\text { Interdisciplinary } \\
\text { Institute }\end{array}$ & Internet & Castelldefels & $25 \mathrm{~km}$ & $\begin{array}{c}\mathrm{C} / \mathrm{Roc} \\
\text { Boronat, } 117\end{array}$ & 2010 \\
\hline Mediapro & Audiovisuales & $\begin{array}{c}\text { Esplugues de } \\
\text { Llobregat }\end{array}$ & $9 \mathrm{~km}$ & $\begin{array}{c}\text { Avinguda } \\
\text { Diagonal, } 177\end{array}$ & 2008 \\
\hline SAGE & Gestión & Sabadell & $28 \mathrm{~km}$ & C/ Josep Pla, 2 & 2012 \\
\hline T-Systems & Tecnología & Madrid & $625 \mathrm{~km}$ & $\begin{array}{c}\text { C/ Sancho de } \\
\text { Ávila, 110-130 }\end{array}$ & 2004 \\
\hline
\end{tabular}

Fuente: elaboración propia

\section{UN PREDOMINIO DE ACTIVIDADES LOCALIZADAS ANTERIORMENTE EN EL CENTRO HIS- TÓRICO DE BARCELONA}

Los distintos traslados descritos anteriormente procedían generalmente de dos áreas de la ciudad. Como se puede ver en la figura 3, se trata de los alrededores del centro histórico de la ciudad (especialmente Via Laietana y Passeig de Gràcia) y del área del Eixample y de la Avinguda Diagonal.

Estas dos áreas tienen la consideración de vías representativas de la tradición económica de Barcelona. En la Via Laietana se localiza mayoritariamente la administración pública, que aún mantiene algunas sedes importantes. En Passeig de Gràcia se implantan algunas sedes empresariales y bancarias. A raíz del aumento del turismo en la ciudad y la transformación del paseo en una zona comercial la mayoría de estas sedes se han transformado, en las últimas décadas, en flagship store y hoteles. En cambio la Avinguda Diagonal era el área que agrupaba, desde los años setenta y hasta la actualidad, la sede corporativa de varias empresas.

Ahora bien, ¿hasta qué punto se ha visto afectada esta distribución tradicional de las sedes corporativas por el traslado hacia el 22@Barcelona? Los resultados muestran que las repercusiones han sido desiguales.

En el área de la Diagonal prácticamente no se ha generado ningún efecto. De los once traslados detectados, siete de los espacios ya estaban ocupados, dos había cambiado su uso por otros servicios y un único local permanecía vacío. En uno de los casos la compañía ya había anunciado el traslado el 22@Barcelona, pero aún no se había llegado a concretar. En consecuencia podemos considerar que la mayoría de locales liberados por los traslados al22@Barcelona han sido ocupados por nuevas compañías. El hecho de que esta sea un área tradicional de sedes corporativas, con una ocupación alta, ha ayudado a la rotación. Una situación similar es la que puede observarse en otros edificios que acogen un volumen importante de oficinas, como el World Trade Center o la Torre Mapfre. En ambos casos, las empresas que han abandonado estos espacios han sido sustituidas sin problemas. 


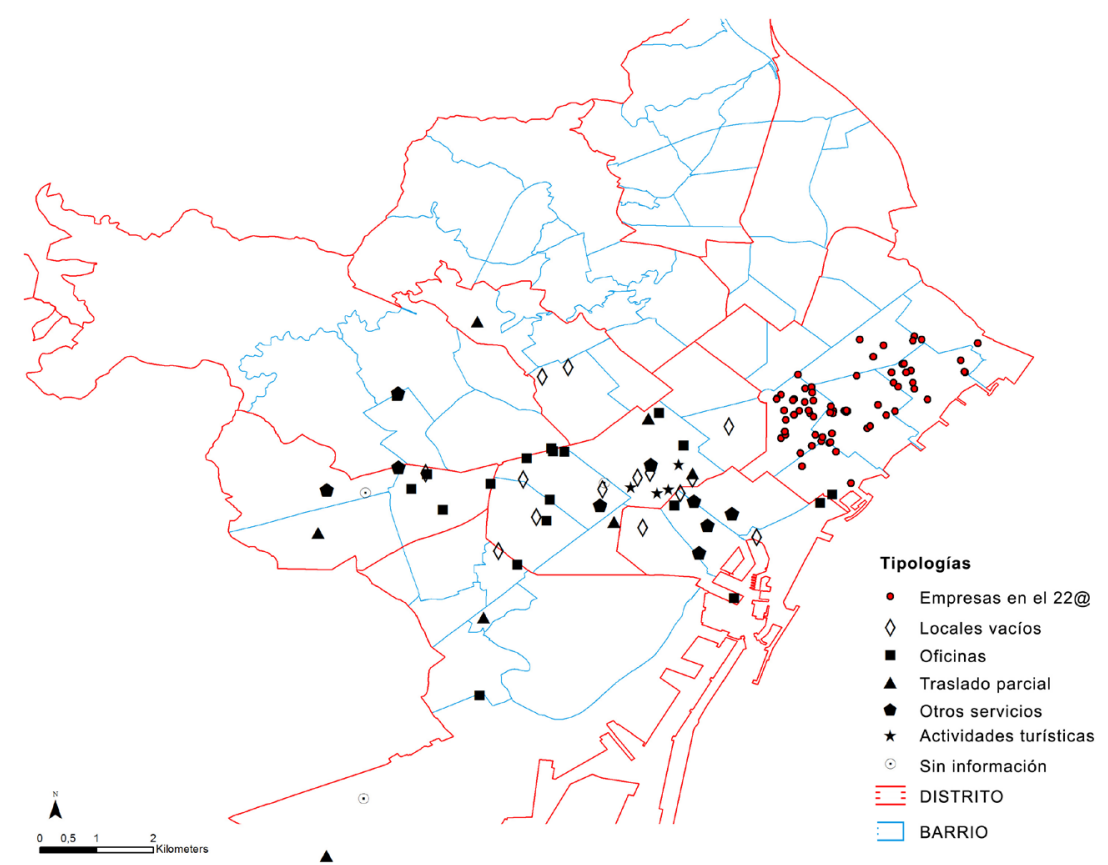

Fuente: Autor. Cartografía base: Ajuntament de Barcelona.

Esta situación de substitución no se da en las otras zonas de la ciudad, donde sí se han observado cambios. Este es el caso muy especial de las antiguas sedes implantadas en el centro histórico. De los ocho casos localizados en este espacio, sólo uno sigue ocupado por oficinas. En cuatro casos los usos han cambiado con la implantación de dos centros de enseñanza universitaria (Elisava y EMAV), un museo y los servicios jurídicos de la Generalidad de Cataluña. Destaca igualmente que en tres de los casos los locales se encuentran vacíos. Los proyectos existentes en estos casos tampoco guardan relación con los usos anteriores. Se trata de la ampliación del museo MACBA y de la probable construcción de dos nuevos equipamientos turísticos en las antiguas sedes de los departamentos de Interior y de Bienestar Social. En consecuencia podemos apuntar que en el caso del centro histórico de Barcelona encontramos un claro empobrecimiento del tejido existente. Las sedes empresariales situadas en esta área han dado paso a estructuras enfocadas a dos sectores ya muy representados en el área: los centros educativos universitarios y los equipamientos enfocados al turismo.

8 A pesar de que las sedes empresariales pueden ser entendidas como servicios administrativos, en la presente clasificación las hemos diferenciado de los otros servicios. Así, en el presente artículo, los "servicios administrativos" se equiparan a oficinas, mientras que por "servicios" entendemos los otros grupos (mantenimiento o transporte entre otros). 
Una situación similar se repite en el área de Passeig de Gracia, especialmente en la zona más cercana al centro histórico. En esta zona hemos detectado trece traslados en dirección a la zona del22@Barcelona. De ellos sólo en dos casos se ha mantenido el uso como oficinas. Es significativo, además, que en ambos casos era la misma compañía que trasladaba parte de la actividad en el 22@Barcelona la que mantenía algunos servicios en la antigua sede. En cuatro casos, que coinciden con los locales más grandes, los espacios han sido ocupados por usos turísticos (hoteles y flagship store). En cuatro casos más los espacios, generalmente situados en una o dos plantas de edificios con usos mixtos, se encuentran vacíos a la espera de nuevos proyectos. En dos casos se han instalado servicios (taller de moda y fotografía). En un caso la antigua oficina se ha transformado en una vivienda privada. Por lo tanto, es significativo que en esta zona, que concentraba hasta no hace muchos años un buen número de sedes bancarias y empresariales, no se haya instalado ninguna nueva compañía en los espacios liberados por los traslados en el22@Barcelona. De este hecho resulta, nuevamente, un empobrecimiento funcional del espacio urbano, y una substitución de usos, en este caso hacia una turistificación evidente del área.

Por último, en los espacios que no forman parte de ninguna de estas tres áreas hemos localizado 18 locales. En seis casos los locales habían sido ocupados por otras compañías. En cinco edificios seguían los mismos inquilinos y en cinco otros locales los espacios se encontraban vacíos. Este último estado es especialmente evidente en los espacios más alejados de los centros tradicionales. En un único caso el local no era accesible y no hemos encontrado información de su uso actual. Destaca también el hecho de que en estas zonas los cambios observados en los espacios centrales de la ciudad prácticamente no se han dado. Significativamente sólo se tiene constancia de un cambio de uso hacia el sector servicios (un despacho de abogados que ocupa la antigua sede de la consultora tecnológica Altran). Igualmente, en esta área, no se tiene constancia de ninguna transformación de antiguas sedes en espacios dedicados a usos turísticos.

\section{PRINCIPALES MOTIVACIONES DE LOS TRASLADOS}

Según la información cualitativa recopilada, en casi un $75 \%$ de los casos analizados el traslado venía justificado en buena medida por aspectos económicos. Los precios de compra y alquiler del 22@Barcelona estaban claramente por debajo de la media de Barcelona cuando se desarrollaron los principales movimientos. Las facilidades para vender la antigua sede para financiar la nueva implantación o el aumento de los precios de los alquileres en las zonas más céntricas contribuyeron a intensificar los traslados hacía el 22@Barcelona.

En el año 2000 el precio medio en el distrito era de $5^{\prime} 7 € / \mathrm{m}^{2} / \mathrm{mes}$, un $5 \%$ más barato que en el conjunto de Barcelona y un $25 \%$ más barato que el distrito más caro (les Corts). Los precios aumentaron en el conjunto de Barcelona hasta 2008, cuando alcanzaron en el distrito los 11 ' $8 € / \mathrm{m}^{2} / \mathrm{mes}$, un precio un 3'3\% más barato que en el conjunto de Barcelona y un 11 '9\% más barato que el distrito más caro. El distrito se había encarecido y había perdido una parte del factor diferencial en comparación al conjunto de la ciudad. A partir de 2008, sin embargo, los precios decaen hasta los $10^{\prime} 5 € / \mathrm{m}^{2} / \mathrm{mes}$ del año 2012 (precios similares a los de 2006). El distrito pasa a ser un $2 \% 8 \%$ más barato que el conjunto de Barcelona, pero un $15 \%$ más barato que los distritos más caros (www.bcn.cat/estadistica). 
No podemos tampoco obviar otra casuística más específica de Barcelona y que pese a no relacionarse directamente con el 22@Barcelona lo ha beneficiado: el turismo. El crecimiento de la llegada de turistas ha supuesto que haya una fuerte presión por parte de servicios o alojamientos dedicados a este colectivo para ocupar determinados espacios. Como hemos visto, un cierto número de estos nuevos hoteles y actividades dirigidas al sector turístico se instalan en antiguas sedes trasladadas al 22@Barcelona. Este hecho, pese a no ser consecuencia del nuevo distrito, supone una mayor competencia para los espacios centrales y mejores precios para la venta, lo que contribuye a facilitar los traslados, como han reconocido aproximadamente la mitad de los entrevistados.

Otro motivo relevante para el traslado, a distancia del primero, fueron los relacionados con las infraestructura. En las zonas ya consolidadas de la ciudad es difícil ampliar el espacio ocupado, por ejemplo para unificar servicios, lo que obliga a cierta fragmentación en múltiples edificios. La disponibilidad de espacio en el 22@Barcelona permite la creación de nuevos edificios que no serían posibles en otros barrios. Muestra de ello es, por ejemplo, la nota de prensa distribuida con motivo del traslado de la cooperativa Abacus:

"El traslado resuelve la carencia de espacio en las actuales oficinas, consecuencia del fuerte crecimiento de los últimos años.".

Además, los nuevos edificios disponen de todos los servicios modernos, a veces difíciles de encontrar en aquellas áreas con construcciones más antiguas. Es posible que sin la actuación del22@Barcelona algunas compañías allí trasladadas hubiesen situado su nueva sede fuera de Barcelona. Un hecho que la nueva disponibilidad de suelo habría evitado.

Finalmente, el último de los aspectos surgidos en las entrevistas para justificar los traslados es el de la proximidad a compañías del mismo sector (clúster tecnológico). Este aspecto es, según los responsables del barrio, una de las fortalezas de la operación. Ahora bien, sorprendentemente, este aspecto no aparece de forma relevante en la información recopilada. Única una empresa ha citado las posibles sinergias como elemento a favor del traslado: la productora Mediapro citó la proximidad con el campus de comunicación de la UPF (y sus estudios) como elemento importante del traslado, pero no como uno fundamental.

\section{VIII. ¿DISTRITO DE LA INNOVACIÓN O CBD DE BAJO COSTE?}

La concretización del proyecto 22@Barcelona muestra, como hemos apuntado a lo largo del texto, algunas debilidades importantes. La creación de nuevos distritos focalizados en una actividad concreta, en el caso de Barcelona las industrias de base tecnológica, puede generar repercusiones en el conjunto de la ciudad. Repercusiones que pueden ser positivas, como aumentar la competitividad de la ciudad, poner a disposición de las distintas compañías nuevos espacios o hacer posible el crecimiento de determinadas actividades.

El 22@Barcelona ha contribuido a generar cambios en estos ámbitos, pero también ha impulsado otras repercusiones. El importante volumen tanto en metros cuadrados como en trabajadores trasladados des de otras áreas de la ciudad hacía el nuevo distrito ha contri-

9 http://www.22barcelona.com/content/view/706/90/lang,es/ (consultado el 23/07/2015) 
buido a modificar el tejido económico de algunos barrios, creando espacios vacíos donde antes había un volumen importante de actividad o impulsando el incremento de actividades turísticas en ciertas áreas centrales. Unos efectos que en buena medida no se habían previsto, confiando en que la fortaleza económica de la ciudad sería suficiente para ocupar los espacios generados por los traslados. Cuando los efectos de la crisis económica fueron evidentes, otras prioridades guiaron la gestión municipal, con lo que prácticamente no han existido políticas municipales que contrarrestaran los efectos descritos.

El análisis de las compañías implantadas en el nuevo distrito permite matizar algunos aspectos del discurso oficial referente al área. Así, el carácter de filial de la mayoría de compañías hacen que la toma de decisiones, incluso las referentes a la implantación en el 22@ Barcelona, se hayan tomado en sedes situadas en otras ciudades. No fue la voluntad de colaboración entre empresas, ni la presencia de centros de formación, de técnicos cualificados o de proveedores los que justificaron la decisión de implantarse en el distrito. La lógica de la mayoría de traslados responde más a operaciones de carácter inmobiliario que industrial. En este sentido el barrio difícilmente puede ser considerado actualmente como un auténtico clúster o un distrito de la creación. Lo anterior no significa que en el futuro no se puedan dar otras sinergias que impulsen un auténtico distrito tecnológico. No obstante, en términos de ciudad, podemos considerar los beneficios de la operación como mitigados.

El proyecto inicial22@Barcelona pretendía, en buena medida, la implantación de nuevas industrias capaces de impulsar el desarrollo de un nuevo central business district (CBD) en la ciudad. Un espacio que en el horizonte 2025-2030 debía unirse a otras áreas en aquel momento en desarrollo, las hoy todavía inconclusas actuaciones de Sagrera y de la zona Fòrum. Los desarrollos urbanísticos de estos tres espacios hubiesen generado en esta área de la ciudad mayor disponibilidad de metros cuadrados que en los espacios tradicionales de implantación de las sedes empresariales (Eixample y Ciutat Vella) (Mur y Clusa, 2011: 51-52). Esta disponibilidad de suelo debería contribuir a atraer nuevas empresas, que impulsarían el desarrollo de un innovador CBD basado en las actividades tecnológicas. Ahora bien, en la actualidad y según los datos analizados, la localización de las compañías en el Poblenou responde básicamente a una lógica de implantación basada en la búsqueda de un CBD de bajo coste.

Son las ganancias derivadas de la operación inmobiliaria las que han justificado la mayoría de traslados. La intensidad de compañías procedentes de áreas con fuertes aumentos de precios y la justificación de los traslados sobre la base económica así lo indicarían. En consecuencia podemos apuntar que no se han cumplido los objetivos planificados de creación de una nueva base económica para la ciudad. No se ha conseguido atraer nueva actividad económica de otros municipios, sino que mayoritariamente se han trasladado compañías ya presentes en la ciudad. Así, el distrito no ha contribuido a fortalecer el tejido económico del conjunto de Barcelona. Más bien ha impulsado un proceso de reordenación de la distribución de las empresas y de creación de un distrito de negocios tradicional del que Barcelona, en buena medida, no disponía.

Algunos autores ya habían apuntado, incluso antes del inicio de la crisis económica, algunas debilidades que podían condicionar el éxito del proyecto 22@Barcelona. Entre ellos destaca Leon (2008: 239) quien señalaba cinco retos que las autoridades debía afrontar para conseguir que el distrito atrajera las sedes de grandes empresas e instituciones internaciona- 
les. Se trataba de conseguir un nivel de capital humano cualificado suficiente, una capacidad emprendedora elevada, disponibilidad de fondos de capital riesgo, presencia de múltiples sedes regionales de grandes empresas capaces de liderar el sistema innovador y mejoras en la conectividad global.

A la luz de los resultados obtenidos se puede considerar que el desarrollo del 22@Barcelona se ha basado, en buena medida, en las empresas ya implantadas en la ciudad, con una escasa capacidad para atraer grandes empresas ubicadas más allá del ámbito metropolitano de Barcelona. Ello ha generado una operación urbanística que como hemos mostrado ha tenido otros efectos importantes sobre el conjunto de la ciudad de Barcelona, ya que ha favorecido que en otras áreas de la ciudad existan grandes edificios sin uso, ha ayudado a impulsar ciertas actividades predominantes como el turismo y ha contribuido a la pérdida de actividad económica en las áreas más alejadas del centro.

Queda claro, en consecuencia, que la creación de nuevos distritos focalizados en una actividad concreta, en el caso de Barcelona las industrias de base tecnológica, pueden contribuir a generar repercusiones en áreas extensas. Unas repercusiones que pueden ser positivas, como el aumento de la competitividad de la ciudad, poner a disposición de las empresas nuevos espacios o bien fomentar el crecimiento de ciertas actividades. Ahora bien, este conjunto de factores no son los únicos que pueden darse. Como se ha mostrado, la relocalización de las distintas empresas puede generar otras actividades, menos esperadas y no siempre positivas.

En las grandes actuaciones urbanas los efectos no se producirán únicamente en las áreas afectadas por la transformación. En este sentido el 22@Barcelona demuestra como las repercusiones de una gran transformación urbana pueden afectar a amplias áreas. Igualmente muestra la necesidad de una buena evaluación de los efectos positivos y negativos de las actuaciones con el fin de conseguir un tejido urbano rico y variado en los espacios urbanos consolidados.

\section{BIBLIOGRAFÍA}

AJUNTAMENT DE BARCELONA (2010): 10 anys de 22@: el districte de la innovació. Barcelona. Ajuntament de Barcelona.

BOIXADER, J. (2005): «La acción colectiva de los agentes urbanos en la transformación de Barcelona» en Scripta Nova. vol. IX, núm. 194-80, 1 de agosto de 2005. Universitat de Barcelona. Disponible en http://www.ub.es/geocrit/sn/sn-194-80.htm.

CARRINCAZEAUX, C. (2000): «La notion de distance dans les analyses empiriques des effets de débordement géographique de la connaissance : une revue de la littérature». R.E.R.U, $\mathrm{n}^{\mathrm{o}} 4,619-642$.

CHECA ARTASU, M. (2007): «Geografías para el patrimonio industrial en España: el caso de Barcelona» en Scripta Nova, vol. XI, núm. 245-32, 1 de agosto de 2007- Universitat de Barcelona. Disponible en http://www.ub.es/geocrit/sn/sn-24532.htm.

DAVEZIES, L. (2006): «L'Île-de-France, une région malade du coeur? ». Urbanisme hors série $29,52-54$.

DOT JUTGLA, E.; CASELLAS, A. y PALLARES-BARBERA M, (2010) «L'ambigüitat de la producció intensiva en coneixement: el nou espai econòmic del Poblenou». Documents d'Anàlisi Geogràfica, vol. 56 (3), 389-408. 
GASTALDI, F. y CAMERIN, F. (2013): «Rigenerazione urbana delle città italiane: occasioni ed opportunità dai processi di dismissione degli immobili pubblici». AREL, $\mathrm{n}^{\circ} 2,95-103$. GAPS (2015): 22@Barcelona.Anàlisi d'activitat económica. Barcelona. Barcelona Activa.

HILLIER, B.A. (2001): «Theory of the city as object, or how the spatial laws mediate the social construction of urban space». International Spaces Syntax Symposium, vol 3, núm. 02.1-02.14. Disponible en eprints.ucl.ac.uk/1029/.

HUTTON, T.A. (2004): «Post-industrialism, Post-modernism and the Reproduction of Vancouver's Central Area: Retheorising the 21st-century City». Urban Studies, vol. 41 (10), 1953-1982.

LEON, N. (2008): «Attract and connect: The 22@ innovation district and the internationalisation of Barcelona business». Innovation: Management, Policy y Practice, vol. 10 (2-3), 235-246.

MUR, S. y CLUSA, J. (2011): «Impacte urbanístic i immobiliari del 22@: el futur 'central business district' de Barcelona». Revista Econòmica de Catalunya, n ${ }^{\circ}$ 64, 31-53.

PAÜL i AGUSTÍ, D. (2014a): «Del Manchester català a districte de la innovació de Barcelona. Els canvis simbòlics del barri de Poblenou de Barcelona» en Scripta Nova, vol. XVIII, núm. 493, 1 de noviembre de 2014. Universitat de Barcelona. Disponible en http://www.ub.edu/geocrit//sn/sn-493/493-14.pdf

PAÜL i AGUSTÍ, D. (2014b): «Repercusiones inesperadas de una transformación urbana ralentizada por la crisis. El retorno de los artistas al distrito creativo 22@Barcelona». Cuadernos Geográficos, vol. 53 (2), 87-102.

PAREJA-EASTAWAY, M. (2010): «Construyendo la Barcelona creativa: nuevos actores, nuevas estrategias». Finisterra, vol. XIV, nº , 90, 133-152.

PARIS, R. (2003): «Essai de mesure des effets externes engendrés par une opération d'aménagement à travers une fonction hédonique des prix fonciers». Revue d'Économie Régionale et Urbaine, $\mathrm{n}^{\circ}$ 4, 671-689.

SABATÉ, J. y TIRONI, M. (2008): «Rankings, creatividad y urbanismo». Revista Eure, vol. $34, n^{\circ} 102,5-23$.

SERDOURA, F.M. (2008): «A emergència de noves centralidades: o caso de Lisboa». Minerva, vol. 5, n ${ }^{\circ} 2,187-196$.

TERRAL, L. y PADEIRO, M. (2013): «La centralité d'affaires parisienne face au desserrement des emplois. Un examen par le biais de la localisation d'activités «témoins» (19932008)». Géographie, économie, Société, vol. 15 (3), 205-237.

TOMSON, B. (2015): «La pression monte». Bruxelles en mouvement, $\mathrm{n}^{\circ}$ 276, 12-13.

TUBA, B.; ALMIRALL, E. y WAREHAM, J. (2013): «A Smart City Initiative: the Case of Barcelona» Journal of the Knowledge Economy. vol. 4, 135-148.

WATKINS, A. R. (2009): «The Dynamics of Urban Economies: Melbourne 1971 to 2006». Urban Studies, vol. 46 (8), 1553-1576. 
